\title{
Application of Minimum-time Optimal Control System in Buck-Boost Bi-linear Converters
}

\author{
Seyed Mohammad Shariatmadar \\ Electrical Engineering Department \\ Naragh Branch, Islamic Azad University \\ Naragh, Iran \\ Shariatmadar@iau-naragh.ac.ir
}

\author{
Seyed Mohammad Javad Jafarian \\ Electrical Engineering Department \\ Shahed University \\ Tehran, Iran \\ mohammad.jafarian@gmail.com
}

\begin{abstract}
In this study, the theory of minimum-time optimal control system in buck-boost bi-linear converters is described, so that output voltage regulation is carried out within minimum time. For this purpose, the Pontryagin's Minimum Principle is applied to find optimal switching level applying minimum-time optimal control rules. The results revealed that by utilizing an optimal switching level instead of classical switching patterns, output voltage regulation will be carried out within minimum time. However, transient energy index of increased overvoltage significantly reduces in order to attain minimum time optimal control in reduced output load. The laboratory results were used in order to verify numerical simulations.
\end{abstract}

Keywords-Buck-boost converter; sliding mode control; minimum-time control; bi-linear converter

\section{INTRODUCTION}

Several methods have been introduced to control DC-to-DC switching converters [1-7]. Common controllers employed in these converters include the PWM traditional controller based on current control or voltage control [1-2]. Design based on the averaging method from the state space based on the small signal method in these controllers will not bring about a proper dynamic performance in large-signal disturbances. Due to the non-linear nature of these converters, nonlinear control methods have been recommended to improve the stability and resistance properties of controller in these systems [4-6]. The sliding mode control technique is one of the modern non-linear methods utilized in variable structure systems such as DC-toDC switching converters [7-8]. Ease of implementation, proper dynamic performance and stability are achieved by the application of this type of nonlinear control technique. Nevertheless, non-fixed and high switching frequency, regulation error of steady state and complicated design are among the difficulties of implementing the sliding mode controller in DC-to-DC switching converters [9].

Non-minimum phase systems encounter certain limitations. This feature in voltage control method leads to complex design and inadequate dynamic response to identify frequency traversing via resonance frequency [1]. Despite the failure to maintain system stability and large signal disturbances, the current control method has faster transient response when compared to voltage control in a similar system. Several methods have been proposed to control boost converter in sliding mode, and especially in the latter years in an effort to enhance their performance [9-16], including PWM constant frequency based on voltage or current control, adaptive sliding mode controller and dual integral sliding level [12-16]. Looking back on these methods, we can conclude that in the majority of these systems, system stability and performance have targeted steady state while static behavior of system have been considered by researchers. Nevertheless, several studies have selected non-linear switching level to enhance system transient performance under sliding mode controllers [17]. In these systems, despite an improvement in system behavior, in contrast to linear switching level, transient response of system in large-signal disturbances do not exhibit proper behavior.

In this study, the Pontryagin's Minimum Principle in buckboost bilinear converters is investigated and regulations of minimum-time optimal control are derived. The optimized closed-loop controller works on output voltage regulation in each output disturbance. Simulation results reveal that the performance of buck-boost time optimal converters exhibited a better transient performance when compared to the sliding mode controller.

\section{INTRODUCTION TO SLIDING MODE CONTROLLER}

Variable nonlinear dynamic system with the timeline below is assumed as follows:

$$
\dot{x}(t)=A x(t)+B_{1} x(t) u(t)+B_{2} V_{i n} u(t)
$$

where $x(t), v u(t), V_{\text {in }}$ are mode vector, disturbance input and input voltage of system respectively. $A, B_{1}, B_{2}$ are also fixed matrices with appropriate dimensions. The initial conditions and balance points in system in $t_{f}$ are assumed as follows:

$$
\begin{aligned}
& x(0)=x_{0} \\
& x\left(t_{f}\right)=x_{f}
\end{aligned}
$$

In addition, restrictions of control input are as follows:

$$
u_{N}<u(t)<u_{P}
$$


where $u_{P}>0$ and $u_{N} \leq 0$ are upper and lower limits of control inputs. In classical systems design of switching level, $S_{s m}(x)$ is a linear combination of system status:

$$
S_{s m}(x)=\beta^{T}\left(x(t)-x\left(t_{f}\right)\right)
$$

Where $\beta$ is the sliding coefficient vector of system. The provisions to reach switching level and sliding on switching level are two sufficient conditions for the implementation of sliding mode controller in one of the customized systems. The first provision is to examine the reaching of system trajectory to switching level which is achieved by one of the following conditions:

$$
\begin{aligned}
& \text { If } x_{P} \in S_{s m}>0 \text { Then } x_{N} \in S_{s m}<0 \\
& \text { If } x_{P} \in S_{s m}<0 \text { Then } x_{N} \in S_{s m}>0
\end{aligned}
$$

Where $x_{P}$ and $x_{N}$ are steady state values of state vector with $u_{P}$ and $u_{N}$ control inputs. The second condition (sliding) will be provided using the following relationship:

$$
\lim _{S_{s m} \rightarrow 0} S_{s m}\left(x_{1}, x_{2}\right) \dot{S}_{s m}\left(x_{1}, x_{2}\right)<0
$$

Where $\left(\dot{S}_{s m}\left(x_{1}, x_{2}\right)\right)$ are time function of switching level function. The sliding coefficients of $\beta$ is selected in (4) in such a way that the condition of (6) will be established. According to (5) and (6), control rule of infinite switching frequency in sliding mode regime is as follows:

$$
u(t)= \begin{cases}u_{p} & S_{s m}(x) \leq 0 \\ u_{N} & S_{s m}(x)>0\end{cases}
$$

The stability of the ystem in second grade systems is provided by trajectory guidance of system towards a stable equilibrium point in sliding mode regime [7].

\section{OPTIMAL CONTROL OF NON-OSCILLATORY DYNAMIC SYSTEMS}

In minimum time problem, we focus on minimizing function as follows:

$$
J(u)=\int_{0}^{t_{f}} d t
$$

We suppose that in (1), the values of $A, B_{1}, B_{2}$ are as follows:

$$
A=\left[\begin{array}{cc}
-\alpha_{1} & 0 \\
0 & -\alpha_{2}
\end{array}\right], B_{1}=\left[\begin{array}{l}
0 \\
0
\end{array}\right], B_{2}=\left[\begin{array}{l}
0 \\
1
\end{array}\right]
$$

Where $a_{1}$ and $a_{2}$ are real eigenvalues of system. The Hamiltonian function of systems (1) with constant values (9) in minimum-time optimal control system is as follows:

$$
\begin{aligned}
& H(x(t), \lambda(t), u(t))= \\
& 1-\lambda_{1}(t) \alpha_{1} x_{1}(t)-\lambda_{2}(t) \alpha_{2} x_{2}(t)-V_{i n} \lambda_{2}(t) u(t)
\end{aligned}
$$

Where $\lambda(t)$ is auxiliary variable of system status which is obtained as follows:

$$
\dot{\lambda}(t)=\left[\begin{array}{cc}
-\alpha_{1} & 0 \\
0 & -\alpha_{2}
\end{array}\right]\left[\begin{array}{l}
\lambda_{1}(t) \\
\lambda_{2}(t)
\end{array}\right]-\left[\begin{array}{l}
0 \\
1
\end{array}\right] u(t) V_{\text {in }}
$$

The optimal condition to apply Pontryagin's Minimum Principle as well as Hamiltonian's Minimum function (10) when compared to $u(t)$ limited control input could be obtained as follows:

$$
u^{*}(t)= \begin{cases}u_{\max } & \lambda_{2}(t)<0 \\ u_{\min } & \lambda_{2}(t)>0\end{cases}
$$

Due to lack of freedom of primary values in status auxiliary variables, minimum-time optimal control command is impossible by this method. On the contrary, according to (1), (3) and (9), system status variables will be obtained as follows:

$$
\begin{gathered}
x_{1}(t)=x_{1}(0) e^{-t \alpha_{1}} \\
x_{2}(t)=\frac{u^{*}(t)}{\alpha_{2}}+e^{-t \alpha_{2}}\left(-u^{*}(t)+x_{2}(0)\right)
\end{gathered}
$$

Where $u^{*}$ could be one and /or zero. Because of the nature of the bang-bang, $u(t)$ command and constant control input, the fastest way to damped fluctuations of system is to place trajectory system on the trajectory leading to equilibrium point. So, the optimal switching level could be obtained by (12)-(13) as reverse time dynamic so that the dynamics of the system could be utilized to move towards the equilibrium point [17]. So, as a result of independent variables' dynamics in the above equations, the optimal switching level could be obtained by eliminating time from (12)-(13) and expressing parameter changes in terms of each other as follows:

$$
x_{2}(t)=\frac{u^{*}(t)}{\alpha_{2}}+e^{-\frac{\alpha_{2}}{\alpha_{1}} \ln \frac{x_{1}\left(t_{f}\right)}{x_{1}(t)}}\left(-u^{*}(t)+x_{2}\left(t_{f}\right)\right)
$$

\section{OPTIMAL CONTROL OF FLUCTUATING DYNAMIC SYSTEMS}

It is assumed that $A, B_{1}, B_{2}$ values in (1) are as follows:

$$
A=\left[\begin{array}{cc}
-\alpha & \omega \\
-\omega & -\alpha
\end{array}\right], B_{1}=\left[\begin{array}{l}
0 \\
0
\end{array}\right], B_{2}=\left[\begin{array}{l}
0 \\
1
\end{array}\right]
$$

Where $a$ and $\omega$ are real and imaginary eigenvalues of system. The Hamiltonian's function in system (1) with constant values (15) in minimum-time optimal control system are as follows:

$$
\begin{aligned}
& H(x(t), \lambda(t), u(t))= \\
& 1-\lambda_{1}(t) \alpha x_{1}(t)-\lambda_{1}(t) \omega x_{2}(t)+ \\
& -\lambda_{2}(t) \alpha x_{2}(t)+\lambda_{2}(t) \omega x_{1}(t)+V_{\text {in }} \lambda_{2}(t) u(t)
\end{aligned}
$$

Where $\lambda(t)$ is the auxiliary variable of system status which is obtained as follows:

$$
\dot{\lambda}(t)=\left[\begin{array}{cc}
\alpha & -\omega \\
\omega & \alpha
\end{array}\right]\left[\begin{array}{l}
\lambda_{1}(t) \\
\lambda_{2}(t)
\end{array}\right]-\left[\begin{array}{l}
0 \\
1
\end{array}\right] u(t) V_{i n}
$$

According to (16) and application of Pontryagin's Minimum Principle as well as Hamiltonian's Minimum 
function (10) compared to limited control input, (11) will be repeated. Due to the nature of the bang-bang, $u^{*}(t)$ command, constant control input and unlimited number of input control status changes, the minimum-time control trajectory for every control input status will be obtained separately:

$$
\begin{gathered}
x_{1}(t)= \\
e^{-\alpha t}\left[x_{1}(0) \cos \omega t+x_{2}(0) \sin (\omega t)+\right. \\
\left.-\frac{u^{*}(t)}{\omega_{n}} \sin (\omega t+\phi)\right)+\frac{\omega}{\omega_{n}^{2}} u^{*}(t) \\
x_{2}(t)= \\
e^{-\alpha t}\left[x_{2}(0) \cos (\omega t)-x_{1}(0) \sin (\omega t)+\right. \\
\left.-\frac{u^{*}(t)}{\omega_{n}} \cos (\omega t+\phi)\right]+\left(\frac{\alpha}{\omega_{n}^{2}}\right) u^{*}(t)
\end{gathered}
$$

While:

$$
\omega_{n}=\sqrt{\omega^{2}+\alpha^{2}}
$$

As earlier mentioned, minimum-time optimal control is obtained by positioning system variables on trajectory resulting from system dynamics and leading them to the point. In fluctuating dynamic systems, with regard to bang-bang property of $u(t)$ control input, Hamiltonian's Minimum function (16) for $\lambda_{2}(t)$ changes as well as dependence of $\lambda_{2}(t)$ status auxiliary function signal on $\omega$ frequency, in minimumtime optimal function, $u(t)$ control input for $\pi / \omega$ maximum time unit will be constant. So, minimum-time switching function to reach ultimate time or equilibrium point of system for lower $\pi / \omega$ time unit (as reverse time) related to moving on trajectory will result from system dynamics.

Thereafter, using the generalized results, minimum-time optimal control will be proved in bilinear systems.

\section{OPTIMAL CONTROL OF BILINEAR SYSTEMS}

Hamiltonian's function (1) with limited control input in minimum-time optimal control is as follows:

$$
\begin{aligned}
& H(x(t), \lambda(t), u(t))= \\
& 1+\lambda^{T}(t)\left(A x(t)+B_{1} x(t) u(t)+B_{2} V_{i n}\right)
\end{aligned}
$$

Where $\lambda(t)$ is the auxiliary variable of system status which is defined as follows:

$$
\begin{aligned}
\dot{\lambda}(t) & =-\frac{\partial H\left(x, \lambda, u_{1}, v, t\right)}{\partial x}= \\
& =-A \lambda(t)-B_{1} \lambda(t) u(t)
\end{aligned}
$$

The optimal condition to apply Pontryagin's Minimum Principle and Hamiltonian's Minimum Function when compared to limited control input could be obtained (17).

In bilinear systems with bang-bang input, depending on the type of control subsystems in each control input (dynamic, fluctuating or dynamic fluctuating), the switching optimum level will be determined.
Figure 1 illustrates the schematic of a buck-boost multiple area converter in nonlinear control of sliding mode. $R_{l}, R_{2}$ and $R_{l}$ are leakage resistance of the inductor, capacitor and output load respectively, $v_{i}$ represents the input voltage, $T_{1}$ and $\mathrm{T}_{2}$ which are defined as $u(t)$ in equations which are open and close at zero value respectively. In this converter, the status variables are defined as follows:

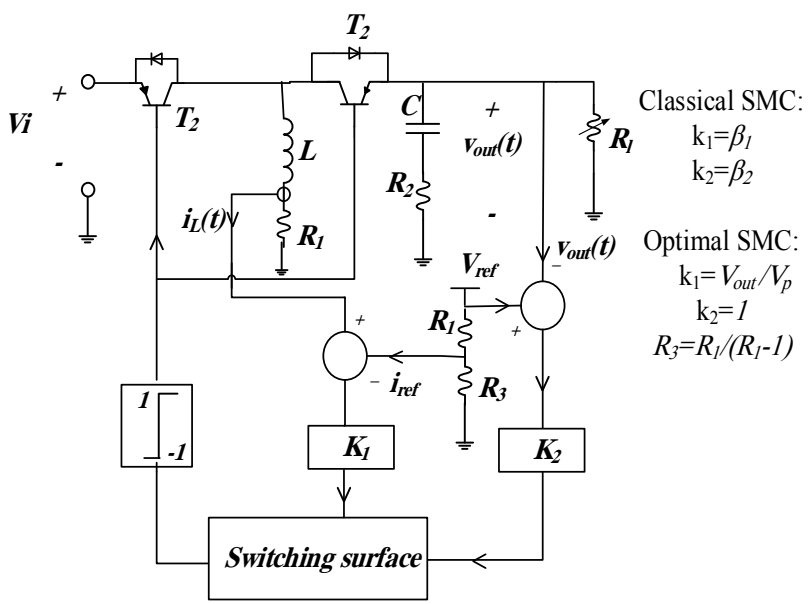

Fig. 1. Buck-boost multiple area converter schematic

VI. MINIMUM-TIME OPTIMAL CONTROL IN BUCK-BOOST MULTIPLE AREA CONVERTER

$$
\left[\begin{array}{l}
x_{1}(t) \\
x_{2}(t)
\end{array}\right]=\left[\begin{array}{cc}
\frac{1}{V_{i}} \sqrt{\frac{L}{C}} & 0 \\
0 & \frac{1}{V_{i}}
\end{array}\right]\left[\begin{array}{l}
i_{l}(t) \\
v_{o}(t)
\end{array}\right]
$$

where $v_{0}(t)$ and $i_{l}(t)$ are capacitor voltage and inductor current, $V_{i}$ is fixed non- regulated input voltage. With normalization of state-space model of buck-boost converter (Figure 1), new equations could be expressed as follows:

$$
\begin{gathered}
\dot{x}_{2}(\tau)=(1-u(\tau)) x_{1}(\tau)+u(\tau) \\
\dot{x}_{1}(\tau)=-(1-u(\tau)) x_{2}(\tau)-\frac{x_{1}(\tau)}{Q}
\end{gathered}
$$

where $Q=R_{l} \sqrt{C / L}$ and $\tau=t / \sqrt{L C}$ are quality coefficient and normalized time variable in buck-boost converter respectively. By comparing (1) and (34), normalized matrix of state space system is displayed as follows:

$$
\mathbf{A}_{1}=\left[\begin{array}{cc}
-\frac{1}{Q} & -1 \\
1 & 0
\end{array}\right] \quad \mathbf{B}_{1}=\left[\begin{array}{cc}
0 & 1 \\
-1 & 0
\end{array}\right] \quad \mathbf{B}_{2}=\left[\begin{array}{l}
0 \\
1
\end{array}\right]
$$

In this model, $u(\tau)$ is controllable input with two 0 and 1 variables. For $u(\tau)=1$ value, system equations will be transformed into non-fluctuating dynamic form (9) and $\alpha_{1}=1 / Q, \quad \alpha_{2}=0$ will be resulted. So, in this case, based on (12) and (13), $x_{1}(\tau)$ and $x_{2}(\tau)$ status variables could be defined as follows: 


$$
\begin{gathered}
x_{1}(\tau)=x_{1}(0) e^{\frac{-\tau}{Q}} \\
x_{2}(\tau)=\tau V_{i}+x_{2}(0)
\end{gathered}
$$

Therefore, minimum-time optimal switching level for $u(\tau)=1$ command will be equal to:

$$
x_{2}(\tau)=x_{2}\left(\tau_{f}\right)+V_{i} e^{-Q \ln \frac{x_{1}(\tau)}{x_{1}\left(\tau_{f}\right)}}
$$

For $u(\tau)=0$, the system equations (25) will be transformed into fluctuating dynamic form (15). Accordingly, $a$ and $\omega$, by calculating eigen values of $A$ matrix will be obtained as $\alpha=\frac{1}{2 Q}, \quad \omega=\frac{\sqrt{1-4 Q^{2}}}{2 Q}$. In this condition, based on (18) and (19),$x_{\text {lnew }}(\tau)$ and $x_{\text {2new }}(\tau)$ status variables could be defined as follows:

$$
\begin{aligned}
& x_{1 \text { new }}(\tau)= \\
& e^{-\alpha \tau}\left[x_{1 \text { new }}(0) \cos (\omega \tau)+x_{2 \text { new }}(0) \sin (\omega \tau)\right] \\
& x_{2 \text { new }}(\tau)= \\
& e^{-\alpha \tau}\left[x_{2 \text { new }}(0) \cos (\omega \tau)-x_{1 \text { new }}(0) \sin (\omega \tau)\right]
\end{aligned}
$$

and:

$$
\left[\begin{array}{l}
x_{1 \text { new }}(t) \\
x_{\text {2new }}(t)
\end{array}\right]=\left[\begin{array}{cc}
0 & \omega \\
1 & -\alpha
\end{array}\right]\left[\begin{array}{l}
x_{1}(t) \\
x_{2}(t)
\end{array}\right]
$$

Figure 2 illustrates the minimum-time optimal switching level function in buck-boost system.

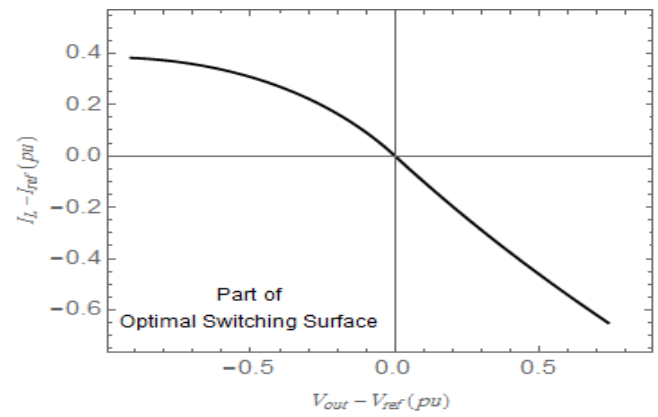

Fig. 2. Optimal switchinglevel function in buck-boost converter

VII. IMPLEMENTATION AND COMPARISON OF MINIMUMTIME OPTIMAL CONTROLLER AND CLASSICAL SLIDING MODE IN BUCK-BOOST MULTIPLE AREA CONVERTER

The switching level for sliding mode converter based on (4), is defined as follows:

$$
\begin{aligned}
& S_{s m}\left(x_{1}, x_{2}\right)= \\
& \beta_{1}\left(x_{1}-V_{r e f}\right)+\beta_{2}\left(x_{2}-I_{r e f}\right)=0
\end{aligned}
$$

while $\beta_{I}=0, \beta 2=1$. Closed loop under reduced output load of increased transient energy index is introduced to compare the transient operation of the two systems as follows:

$$
j(t)=\int_{0}^{t}\left(v_{\text {out }}(t)-v_{\text {ref }}\right)^{2} d t
$$

The simulation results of output voltage and inductor current in buck-boost converter under reduction of load from $R_{l}=5 \Omega$ to $R_{l}=15 \Omega$ in classical and minimum-time optimal systems are illustrated in Figures 3-4. The Buck-boost parameters are shown in Table I.
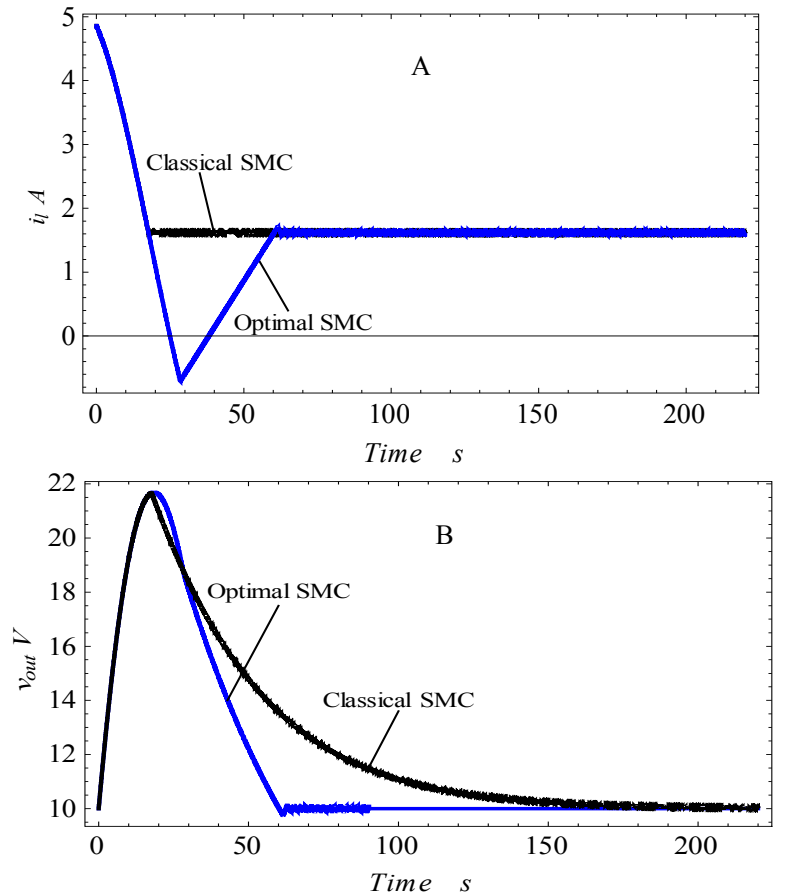

Fig. 3. Fig. 3. Buck-boost multi area converter to reduce output load from 5 to $15 \Omega$. A) output voltage B) inductor current

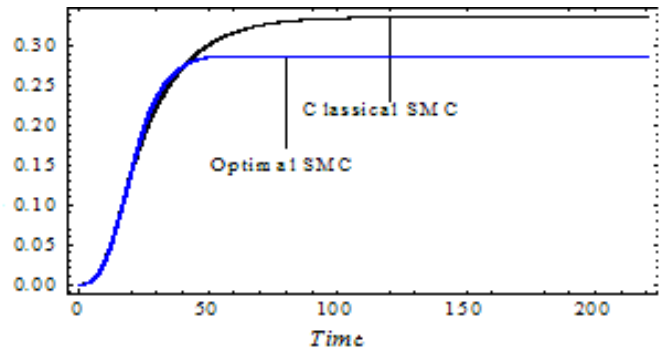

Fig. 4. Increased energy index of classical multi area converter and optimal buck-boost minimum-time systems under reduction of load from 5 to $15 \Omega$.

The results indicated that despite the equality of maximum voltage amplitude of current in minimum-time optimal and classical sliding mode systems, the disturbance in minimumtime optimal systems was omitted within time less than similar classical systems. The increased energy index induced from output voltage (33) in minimum-time optimal systems is lower 
than classical systems (Figure 4). Figure 5 shows the experimental setup that provided for the test. Figure 6 illustrates the applied results of reduced load which confirms the findings of analytical simulation analysis. In second condition, system performance under increased load from $R_{l}=15 \Omega$ to $R_{l}=5 \Omega$ in classical as well as minimum-time optimal systems has been compared and analyzed. The output voltage simulation results and inductor current with $V_{P}=7 \mathrm{~V}$, $V_{N}=0$ and $V_{\text {ref }}=10$ under increased load from $R_{l}=5 \Omega$ to $R_{l}=15 \Omega$ are illustrated in Figure 7. According to this figure, we could say that inductor current needs to save more energy so as to achieve output voltage regulation within minimum time.

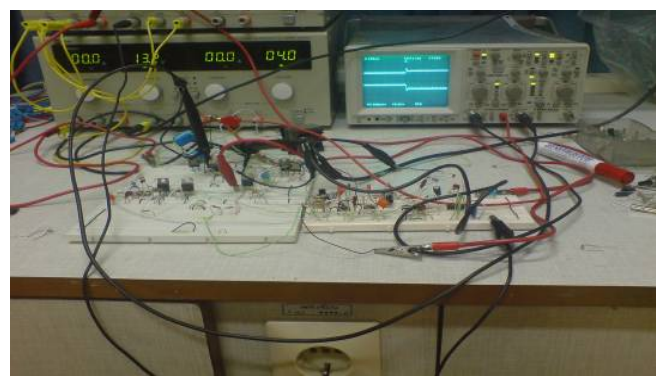

Fig. 5. Experimental Setup

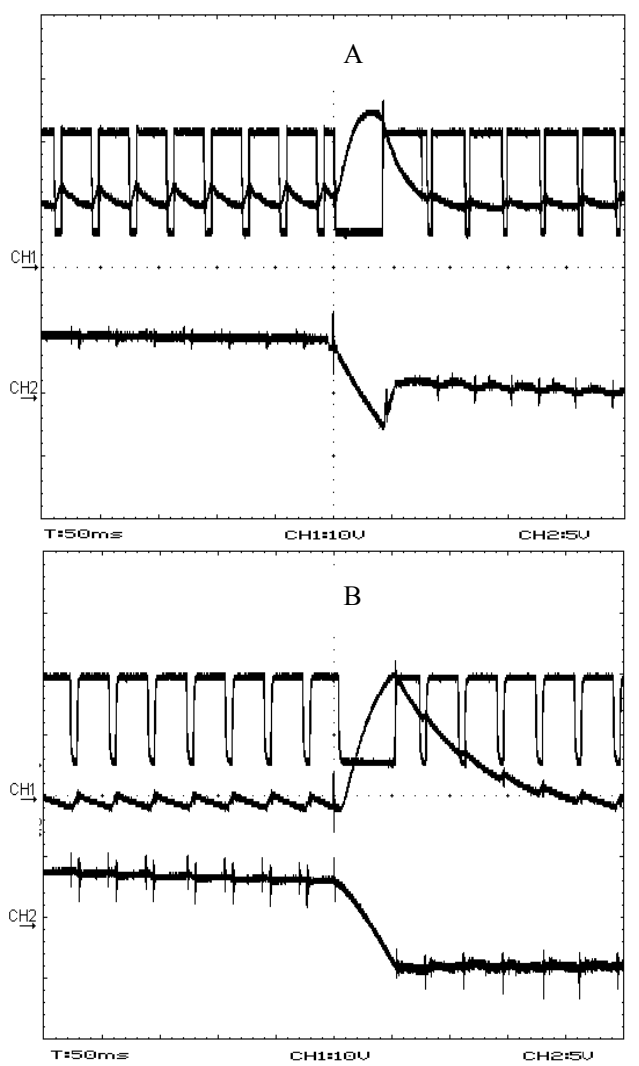

Fig. 6. Buck-boost multi area converter in reduced load from 5 to $15 \Omega$. A) Schematic, B) Optimal controller.

Despite the access to minimum regulation of output voltage in increased output load, voltage drop amplitude in minimum- time optimal systems is larger than similar classical sliding mode systems. So, application of minimum-time optimal control in buck-boost multi area converter enables us to remove disturbances in the least possible time and energy. In this situation, converter switching is a function of status variables (output voltage and inductor current) as though it will reach the maximum value simultaneously in output phase voltage phase and inductor current. So, the minimum-time optimal controller enhances the system dynamic performance and decreases the transient parameter of output overvoltage. $V_{N}$

TABLE I. BUCK- BOOST PARAMETERS

\begin{tabular}{|c|c|c|}
\hline Description & Parameter & Nominal Value \\
\hline Positive voltage & $V_{P}$ & $7 \mathrm{~V}$ \\
\hline Negative voltage & $V_{N}$ & $\{0$ or -7$\}$ \\
\hline Capacitance & $\mathrm{C}$ & $3.4 \mu \mathrm{F}$ \\
\hline Inductance & $\mathrm{L}$ & $96 \mu \mathrm{H}$ \\
\hline Load resistance & $\mathrm{R}$ & $\{11 \Omega, 15 \Omega\}$ \\
\hline Desired output voltage & $V_{\text {ref }}$ & $10 \mathrm{~V}$ \\
\hline $\begin{array}{c}\text { Leakage resistance } \\
\text { inductor }\end{array}$ & $R_{1}$ & $0.2 \Omega$ \\
\hline $\begin{array}{c}\text { Leakage resistance } \\
\text { capacitor }\end{array}$ & $R_{2}$ & $10^{-8} \Omega$ \\
\hline \multicolumn{2}{|c}{} \\
\hline
\end{tabular}
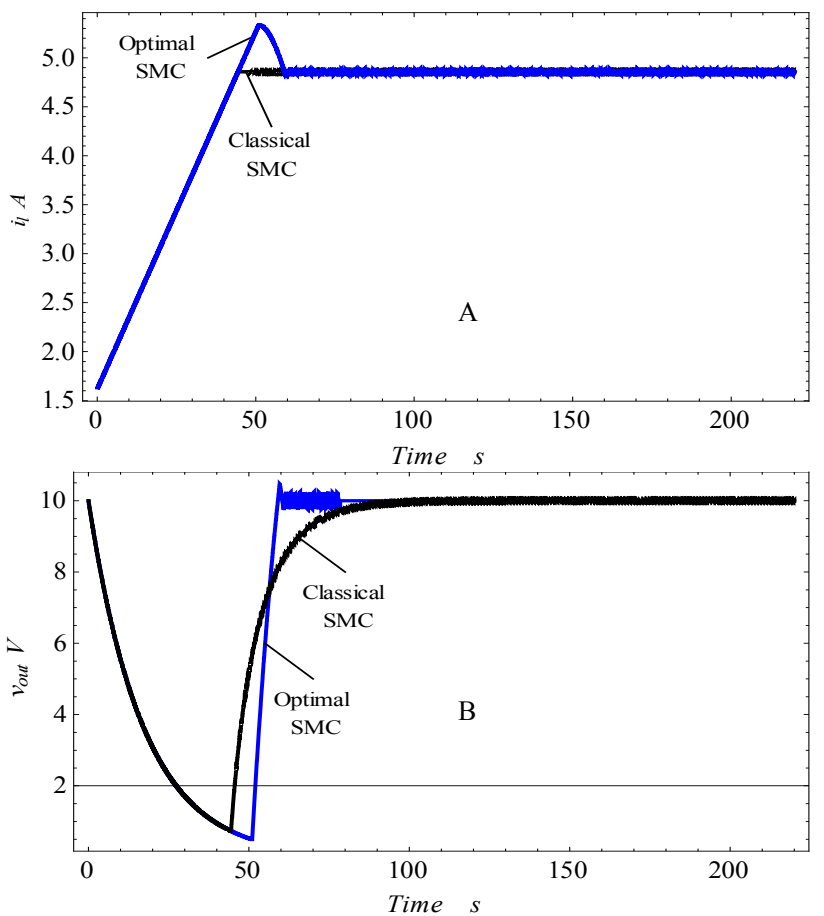

Fig. 7. Classical converter and buck-boost minimum-time optimal systems in increased output load from 15 to $5 \Omega$. A) Output voltage, B) Inductor current

\section{CONCLUSION}

The present study examined the modeling and designing of minimum-time optimal controller of sliding mode in buckboost multi area converter systems. Findings were also examined and analyzed. The results show that the application of switching level instead of classic model causes the output voltage regulation to be implemented within minimum time. 
However, the transient energy index of increased voltage in minimum-time optimal controller significantly decreases. By substituting optimal instead of classical switching surface, the output voltage could be regulated in minimum time. In addition, transient over voltage energy index in optimal buckboost SMC for load step decreased was effectively reduced.

\section{REFERENCES}

[1] H. P. Nabi, P. Dadashi, A. Shoulaie, "A novel structure for vector control of symmetrical six-phase induction machines with three current sensors", Engineering, Technology \& Applied Science Research,Vol. 1, No. 2, pp. 23-29, 2011

[2] R. Jadeja, A. Ved, S. Chauhan, "An Investigation on the performance of random PWM controlled converters", Engineering, Technology \& Applied Science Research,Vol. 5, No. 6, pp. 876-884, 2015

[3] P. Swarnkar, S. Jain, R. K. Nema, "Effect of adaptation gain in model reference adaptive controlled second order system", Engineering, Technology \& Applied Science Research,Vo.1, No. 3, pp. 70-75, 2011

[4] S. Hiti, D. Borojevic, "Robust nonlinear control for the boost converter," IEEE Transaction on Power Electronics, Vol. 10, No. 6, pp. 651-658, 1995

[5] M. K. Kazimierczuk, A. Massarini, "Feedforward control dynamic of DC/DC PWM boost converter", IEEE Transactions on Circuits and Systems-I: Fundamental Theory and Applications, Vol. 44, No.2, pp. 143-149, 1997

[6] M. K. Kazimierczuk, L. A. Starman, "Dynamic performance of PWM DC/DC boost converter with input voltage feedforward control," IEEE Transactions on Circuits and Systems-I: Fundamental Theory and Applications, Vol. 46, No. 12, pp. 1473-1481, 1999

[7] V. Utkin, J. Guldner, J. X. Shi, Sliding Mode Control in Electromechanical Systems, Taylor and Francis, U.K., 1999

[8] T. L. Skvarenina, The Power Electronics Handbook, CRC Press, Boca Raton, FL, 2002

[9] L. Gavini, A. Izadian, L. Li, "A parallel compensation approach in controls of buck-boost converters", IECON 37th Annual Conference on IEEE Industrial Electronics Society, Australia, November 7-10, 2011

[10] S. C. Tan, Y. M. Lai, K. H. Cheung, C. K. Tse, "A unified approach to the design of PWM based sliding mode voltage controller for basic DCDC converters in continuous conduction mode", IEEE Trans. Circuit and Systems, Vol. 53, No. 8, pp. 1816-1827, 2006

[11] Y. B. Shtessel, A. S. I. Zinober, I. A. Shkolnikov, "Sliding mode control of boost and buck-boost power converters using method of stable system centre", Automatica, Vol. 39, No. 6, pp. 1061-1067, 2003

[12] Z. Iwai, I. Mizumoto, L. Liu, S. L. Shah, H. Jiang, "Adaptive stable PID controllerwith parallel feedforward compensator", 9th International
Conference on Control Automation Robotics and Vision (2006 ICARCV), Singapore, 5-8 December, 2006

[13] S. C. Tan, Y. M. Lai, M. K. H. Cheung, C. K. Tse, "Indirect sliding mode control of power converters via double integral sliding surface", IEEE Trans. Power Electron., Vol. 23, No. 2, pp. 600-611, 2008

[14] H. El Fadil, F. Giri, H. Ouadi, "Adaptive sliding mode control of PWM boost dc-dc converters", 2006 IEEE Computer Aided Control System Design, 2006 IEEE International Conference on Control Applications, 2006 IEEE International Symposium on Intelligent Control, Munich, Germany, October 4-6, 2006

[15] V. M. Nguyen, C. Q. Lee, "Indirect implementations of sliding mode control law in buck-type converters", IEEE Appl. Power Electron. Conf. Expo (APEC), Vol. 1, pp. 111-115, 1996

[16] J. Mahdavi, A. Emadi, H. A. Toliyat, "Application of state space averaging method to sliding mode control of PWM DC/DC converters", IEEE Conference on Industrial Electronics Society (IECON), pp. 157162,1999

[17] M. J. Jafarian, J. Nazarzadeh,“Time-optimal sliding-mode control for multi-quadrant buck converters", IET Power Electronics, Vol. 4, No. 1, pp. 143-146, 2011 